\title{
Wormlike Chain Parameters of Cellulose and Cellulose Derivatives
}

\author{
Masatoshi SAITO \\ Textile Research Laboratory, Asahi Chemical Industry Co. Ltd., \\ Takatsuki, Osaka 569, Japan
}

(Received October 14, 1982)

\begin{abstract}
In order to estimate equilibrium chain rigidity, literature data for 12 systems of cellulose and its derivatives and solvents were analyzed on the basis of a wormlike chain model. The persistence length $q$ was calculated by four methods. The $q$ values at the unperturbed state $\left(q_{\mathrm{BD}}^{0}\right)$ agreed closely with those derived using the pearl necklace model. The $q$ values evaluated by the Yamakawa-Fujii (YF) theory were smaller than those obtained by the Benoit-Doty equation $\left(q_{\mathrm{BD}}\right)$; this discrepancy was considered due to the neglect of the partially free draining effect in the YF theory. With increasing solvent polarity, the polymer chain becomes stiffer owing to an increase in solvent-polymer interaction. Most of $q_{\mathrm{BD}}^{0}$ for cellulose derivatives were in the range between $3 \times 10^{-7}$ and $8 \times 10^{-7} \mathrm{~cm}$, except that for cellulose nitrate. Cellulose and its derivatives may be considered semiflexible.

KEY WORDS Cellulose / Cellulose Derivatives / Wormlike Chain Model / Excluded Volume Effect / Persistence Length / Partially Free Draining Effect /
\end{abstract}

A number of studies have been carried out on the dilute solution properties of cellulose and its derivatives. In these studies, the molecular parameters of the polymers have been mainly estimated using the pearl necklace model. Kamide and Miyazaki ${ }^{1}$ concluded from a systematic analysis of experimental data that the conformation parameter $\sigma$ for cellulose and its derivatives lies between $2.8-7.5$ and that the characteristic ratio $C_{\infty}$ is in the range 19-115. These unexpectedly large values of $\sigma$ and $C_{\infty}$ suggest that the molecules of cellulose and cellulose derivatives behave as a semiflexible or inflexible chain. For extremely inflexible polymers, analysis of dilute solution properties by the pearl necklace model becomes theoretically inadequate. Thus, the applicability of this model to cellulose and its derivatives in solution should be carefully examined.

The wormlike chain was first proposed by Kratky and Porod ${ }^{2}$ as a model for chain molecules with large equilibrium rigidity, which is conventionally expressed in terms of the persistence length $q$. The relationship between $q$ and root mean-square radius of gyration $\left\langle S^{2}\right\rangle^{1 / 2}$ of a wormlike chain was derived by Benoit and Doty (BD), ${ }^{3}$ and equations for the limiting viscosity number $[\eta]$ and the sedimentation coefficient $s$ of the same chain were obtained by Hearst, ${ }^{4}$ Peterlin, ${ }^{5}$ Hearst and Stockmayer (HS), ${ }^{6}$ Ullman, ${ }^{7,8}$ and Yamakawa and Fujii (YF). ${ }^{9,10}$ The $q$ values of many inflexible macromolecules, such as DNA, ${ }^{7,10}$ and poly(hexyl isocyanate), ${ }^{11}$ were successfully determined by analyzing data for $\left\langle S^{2}\right\rangle^{1 / 2},[\eta]$, and $s$ by these theories.

The excluded-volume effect can be incorporated in the BD theory for $\left\langle S^{2}\right\rangle^{1 / 2}$, but not easily in the hydrodynamic theories for $[\eta]$ and $s$.

Another method for estimating $q$ is to use the unperturbed chain dimensions $A$ at the coil limit. Penzel and Schulz ${ }^{12}$ estimated $q$ for cellulose nitrate in acetone by this method, but the $q$ value obtained corresponded to infinite molecular weight. Although $q$ is expected to depend on the molecular weight of cellulose derivatives, the $q$ value at finite molecular weight cannot be determined by this method.

In order to compare the equilibrium rigidities of cellulose and its derivatives with those of typical inflexible macromolecules and examine the validity 
of the methods based on the pearl necklace model for cellulose and its derivatives, an attempt was made to calculate $q$, the segment diameter $d$, and the expansion factor $\alpha_{\mathrm{s}}$ of cellulose and its derivatives in solution.

\section{THEORETICAL BACKGROUND}

The persistence length $q$ may be evaluated using any one of the following four methods.

\section{Method Using Light Scattering Data-1 (Benoit- Doty's Method)}

Benoit-Doty's expression ${ }^{3}$ for the unperturbed radius of gyration $\left\langle S^{2}\right\rangle_{0}$ of a monodisperse wormlike chain (Figure 1a) is

$$
\begin{aligned}
\left\langle S^{2}\right\rangle_{0}= & q M /\left(3 M_{\mathrm{L}}\right)-q^{2}+2 q^{3} M_{\mathrm{L}} / M \\
& -2\left(\frac{q^{4} M_{\mathrm{L}}^{2}}{M^{2}}\right)\left[1-\exp \left(-M / q M_{\mathrm{L}}\right)\right]
\end{aligned}
$$

where

$$
\begin{aligned}
M_{\mathrm{L}} & =M / L \\
L & =N l
\end{aligned}
$$

Here, $M$ is the molecular weight, $L$, the contour length, $N$, the number of monomer units, and $l$, the length of a monomer unit projected on the contour axis of the chain. According to Maeda et al. ${ }^{13} L$ for a wormlike chain may be taken as the total length of an extended chain, in which all repeating units take on the trans-conformation with the valence angle fixed constant. In our study, the value of $l$ was taken to be $5.15 \times 10^{-8} \mathrm{~cm}$, which is one half the length of the $c$ axis (parallel to the chain axis) of a unit cell in the cellulose II crystal. ${ }^{14}$

Actual polymers are not monodisperse. Therefore, eq 1 was extended to polymers having Schulz-Zimm type molecular weight distributions. The result is

$$
\begin{aligned}
\left\langle S^{2}\right\rangle_{0, z} & =q^{2}\left[\frac{M_{w}\left(h^{\prime}+2\right)}{3 q M_{\mathrm{L}}\left(h^{\prime}+1\right)}-1+\frac{2 q M_{\mathrm{L}}}{M_{w}}\right. \\
& \left.-\frac{2 q^{2} M_{\mathrm{L}}{ }^{2}\left(h^{\prime}+1\right)}{h^{\prime} M_{w}{ }^{2}}\left\{1-\left(\frac{q M_{\mathrm{L}}\left(h^{\prime}+1\right)}{q M_{\mathrm{L}}\left(h^{\prime}+1\right)+M_{w}}\right)^{h^{\prime}}\right\}\right]
\end{aligned}
$$

where $h^{\prime-1}=M_{w} / M_{n}-1, M_{w}$ and $M_{n}$ are the weight- and number-average molecular weights, and $\left\langle S^{2}\right\rangle_{0, z}$ is the $z$-average unperturbed radius of a) Worml ike chain model

(kratky-Porod)

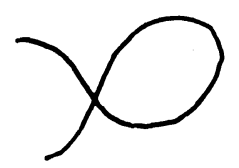

b) Worml ike touched beads model (Hearst, Hearst-Stockmayer, Yamakawa, Stockmayer)

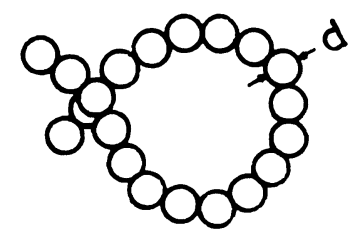

C) Worml ike cylinder model (Ul lman, Yamakawa-Fuji i)

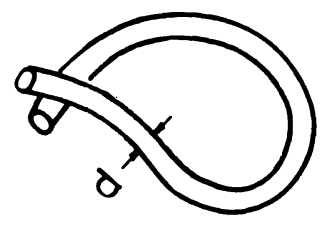

Figure 1. Schematic representation of various wormlike chain models. $d$ is the diameter of a wormlike touched bead (b) or wormlike cylinder (c).

gyration. $q$ can be evaluated by putting data of $\left\langle S^{2}\right\rangle_{z}$ and $M_{w}$ from light scattering measurements and $h^{\prime}$ into eq 4 . We denote this $q$ by $q_{\mathrm{BD}}$.

By incorporating the excluded-volume effect in the wormlike touched-bead chain (see Figure 1b), Yamakawa and Stockmayer (YS) ${ }^{15}$ derived the penetration function $\psi$ as a function of the excluded-volume effect parameter $z$ in the form

$$
\begin{aligned}
\psi & \equiv\left[A_{2} M_{w}{ }^{2} /\left(4 \pi^{3 / 2} N_{\mathrm{A}}\left\langle S^{2}\right\rangle_{w}^{3 / 2}\right)\right] \\
& =\left(\left\langle S^{2}\right\rangle_{0} /\left\langle S^{2}\right\rangle_{0, \infty}\right)^{-3 / 2}[\bar{z} h(\bar{z})]
\end{aligned}
$$

with

$$
\begin{gathered}
\left\langle S^{2}\right\rangle_{0, \infty}=q M /\left(3 M_{\mathrm{L}}\right) \\
\bar{z}=z / \alpha_{\mathrm{s}}{ }^{3}
\end{gathered}
$$




$$
h(\bar{z})=1-Q(L, q, d) \bar{z}+\cdots
$$

Here, $A_{2}$ is the second virial coefficient, $N_{\mathrm{A}}$ Avogadro's constant, $\alpha_{\mathrm{s}}\left(\equiv\left\langle S^{2}\right\rangle^{1 / 2} /\left\langle S^{2}\right\rangle_{0}^{1 / 2}\right)$ the expansion factor, and $Q(L, q, d)$ a function of $L, q$, and the diameter $d$ of a bead. Yamakawa and Stockmayer also derived the relation between $\alpha_{\mathrm{s}}$ and $z$

$$
\alpha_{\mathrm{s}}^{2}=1+(67 / 70) K(L, q) z+\cdots
$$

where $K(L, q)$ is a function of $L$ and $q$. For $\alpha_{\mathrm{s}}$ close to unity, Yamakawa and Stockmayer employed a fifth-power law type closed expression..$^{15}$ On the other hand, Kamide and his coworkers ${ }^{1,16-19}$ evaluated $\alpha_{\mathrm{s}}$ of cellulose and its derivatives using the penetration function of the third-power law type proposed for the pearl necklace model. In order to compare these $\alpha_{\mathrm{s}}$ values with those based on the wormlike touched-bead model, the following closed expression of the Fixman type ${ }^{20}$ was derived ${ }^{21}$ :

$$
\alpha_{\mathrm{s}}^{3}-1=(3 / 2)[67 K(L, q) / 70] z
$$

We derived a closed form of $h(\bar{z})$ as follows ${ }^{21}$ :

$$
\bar{z} h(\bar{z})=\frac{1-[1+(3.903 / 2.865) Q \bar{z}]^{-0.478}}{(1.828 / 2.865) Q}
$$

Equation 11 corresponds to the equation proposed by Kurata-Fukatsu-Sotobayashi-Yamakawa $(\text { KFSY-II) })^{22}$ for the pearl necklace model. We determined $q$ and $\alpha_{\mathrm{s}}$ numerically from eq 10 and 11 . The resulting values are denoted by $q_{\mathrm{BD}}^{0}$ and $\alpha_{\mathrm{s}, 3}^{w}$.

\section{Method Using Light Scattering Data-2 (Gaussian \\ Chain Approximation Method)}

For indefinitely large $M /\left(q M_{\mathrm{L}}\right)$, an unperturbed polymeric chain can be regarded as Gaussian. For this chain, $q$ is related to the unperturbed chain dimension $A_{\infty}$ at infinite $M_{w}$ by

$$
q=M_{\mathrm{L}} A_{\infty}^{2} / 2
$$

The $q$ value estimated by eq 12 is denoted as $q_{\mathrm{CL}}^{0}$.

We determined $A$ for each cellulose derivative, using method $2 \mathrm{~B}$ proposed by Kamide and Miyazaki, ${ }^{1}$ in which $A$ is given by $\left(6\left\langle S^{2}\right\rangle /\left(\alpha_{s}{ }^{2} M\right)\right)^{1 / 2}$ and $\alpha_{\mathrm{s}}$ is evaluated from $\psi$ with the aid of KFSY-II. This value of $\alpha_{s}$ is designated by $\alpha_{s, 3}^{p}$. The $A$ values of cellulose derivatives often depend significantly on molecular weight in the low molecular weight region. In such cases, we approximated $A_{\infty}$ by $A$ of the highest molecular weight samples.
Method Using Limiting Viscosity Number (Yamakawa and Fujii's Method)

Neglecting the draining term in the KirkwoodRiseman integral equation, ${ }^{23}$ Yamakawa and Fujii ${ }^{10}$ derived an expression of $[\eta]$ for unperturbed wormlike cylinders (Figure 1c), which reads

$$
[\eta]=\Phi \cdot\left(2 q / M_{\mathrm{L}}\right)^{3 / 2} M^{1 / 2}
$$

where $\Phi$ is a function of $q, L$, and the diameter of the cylinder $d$ (designated by $d_{\mathrm{h}}^{w}$ ). We determined $q$, referred to as $q_{\mathrm{YF}}$, for each polymer sample.

\section{Evaluation of the Diameter $d_{\mathrm{h}}^{w}$}

The YF theory requires the value of $d_{\mathrm{h}}^{w}$ to determine $q$. Korneeva et al. ${ }^{24}$ estimated $d_{\mathrm{h}}^{w}$ of certain cellulose esters using the Hearst-Stockmayer (HS) theory. ${ }^{6}$ As Yamakawa and Fujii pointed out, ${ }^{9}$ the HS theory contains a mathematical error when converting the summation type equation to an integral equation and hence yields an inaccurate $d_{\mathrm{h}}^{w}$. The hydrodynamic segment diameter estimated from the draining parameter $X$ for the pearl necklace model (designated as $d_{\mathrm{h}}^{p}$; the superscript $p$ means the pearl necklace model and the subscript $h$ a hydrodynamic segment) varies markedly with the chosen length of a hydrodynamic segment, $a_{\mathrm{h}}^{p}$. If $a_{\mathrm{h}}^{p}$ is equal to the length of a statistical segment $a_{\mathrm{s}}^{p}$ in the pearl necklace chain, we obtain $d_{\mathrm{h}}^{p} \simeq 3.5 \times 10^{-7}$ $\mathrm{cm}^{19}$ for cellulose acetate (CA) (degree of substitution, $\mathrm{DS}=2.46$, hereafter the number in parenthesis denotes a DS value) in $N, N$-dimethylacetamide (DMAc). This $d_{\mathrm{h}}^{p}$ is too large to be attributed to the solvation alone. Hence, we determine $d_{\mathrm{h}}^{w}$ on the following assumptions. (1) The hydrodynamic segment lengths $\left(a_{\mathrm{h}}^{p}\right.$ and $\left.a_{\mathrm{h}}^{w}\right)$ in the pearl necklace and wormlike chain models are proportional to the statistical segment lengths $a_{\mathrm{s}}^{p}$ and $a_{\mathrm{s}}^{w}$ in the corresponding models:

$$
\begin{array}{ccc}
a_{\mathrm{h}}^{p}=\lambda a_{\mathrm{s}}^{p}, & \text { and } & a_{\mathrm{h}}^{w}=\lambda a_{\mathrm{s}}^{w} \\
M_{0 \mathrm{~h}}^{p}=\lambda M_{0 \mathrm{~s}}^{p}, & \text { and } & M_{0 \mathrm{~h}}^{w}=\lambda M_{0 \mathrm{~s}}^{w}
\end{array}
$$

where $\lambda$ is a proportionality constant, and $M_{0 \mathrm{~h}}$ and $M_{0 \mathrm{~s}}$ are the molecular weights of the hydrodynamic and statistical segments, respectively. (2) $d_{\mathrm{h}}^{w}$ of the wormlike cylinder is equal to the diameter $d_{\mathrm{h}}^{p}$ of beads consisting of a pearl necklace chain:

$$
d_{\mathrm{h}}^{w}=d_{\mathrm{h}}^{p}=d_{\mathrm{h}}
$$

$a_{\mathrm{s}}^{p}$ and $M_{\mathrm{Os}}^{p}$ are given as functions of $A$ and $M_{\mathrm{L}}$ in 
eq 4 and 5 of ref 19.

Now, $X$ is expressed by ${ }^{25}$

$$
X=\zeta_{\mathrm{p}}\left(M / M_{\mathrm{Oh}}^{p}\right)^{1 / 2} /\left(6^{1 / 2} \pi^{3 / 2} a_{\mathrm{h}}^{p} \eta_{0}\right)
$$

where $\zeta_{\mathrm{p}}$ is the friction constant characteristic of the fluid and the segment and $\eta_{0}$ is the viscosity of the solvent. The friction constant $\zeta_{p}$ obeys the Stokes law, i.e.,

$$
\zeta_{\mathrm{p}}=3 \pi \eta_{0} d_{\mathrm{h}}
$$

With eq 14-17 and eq 4,5 of ref $19, d_{\mathrm{h}}$ can be expressed as follows:

$$
d_{\mathrm{h}}=\lambda^{3 / 2}(2 \pi / 3)^{1 / 2}\left(A^{3} M_{\mathrm{L}}{ }^{2}\right) X M^{-1 / 2}
$$

The friction constant $\zeta_{\mathrm{w}}$ per unit length of the wormlike cylinder is assumed to be equal to $\zeta_{\mathrm{p}}$ per unit length of the pearl necklace chain, i.e.,

$$
\zeta_{\mathrm{w}}=\zeta_{\mathrm{p}} / a_{\mathrm{h}}^{p}
$$

Ullman ${ }^{7}$ derived a relation between $\zeta_{\mathrm{w}}$ and $[\eta]$ for the wormlike cylinder with a finite cross section (see Figure 1c), i.e.,

$$
[\eta]=\left(\frac{\sqrt{2} \pi N_{\mathrm{A}} q^{2} k}{100 M_{\mathrm{L}}}\right) \sum_{i=1}^{n} W_{i} \phi\left(x_{i}, x_{i}\right)
$$

with

$$
k=\sqrt{2} \zeta_{\mathrm{w}} /\left(12 \pi \eta_{0}\right)
$$

Here, $W_{i}$ is the weight associated with variable $x_{i}$ in the Gaussian quadrature formulas; $k$ is a hydrodynamic interaction parameter corresponding to the draining parameter $X$ for the pearl necklace chain, and $\phi\left(x_{i}, x_{i}\right)$ is a dimensionless quantity proportional to the torque of the hydrodynamic force acting on a point of the wormlike cylinder. The equations in the Appendix of ref 7 have been corrected for many typographical errors. ${ }^{26}$ Using the Gaussian quadrature with $n$ (in eq 20) $=20$ and $m$ (in eq R3 of ref 26) $=80$ (hereafter referred to as 20-80 GQ), Ullman ${ }^{7}$ calculated $[\eta]$ in eq 20 for $M_{\mathrm{L}}=1.95 \times 10^{10} \mathrm{~cm}^{-1}$. In order to check the reliability of our calculation programs, we calculated $[\eta]$ from eq 20 and 20-80 GQ, using the same values of $M_{\mathrm{L}}$ and $d_{\mathrm{h}}$ as chosen by Ullman. The calculated $[\eta]$ agreed with Ullman's within $0.5 \%$ relative error for $q=1.56 \times 10^{-6} \mathrm{~cm}$. In general, in the ranges $q \leq 6.25 \times 10^{-6} \mathrm{~cm}, L \leq 4 \times 10^{-4} \mathrm{~cm}$, and $k \geq 1 \times 10^{-2}$, our $[\eta]$ values were in agreement with Ullman's within $2 \%$ relative error.

Substitution of eq $17-19$ into eq 21 gives

$$
k=\sqrt{\pi \lambda / 12} X A M_{\mathrm{L}} M^{-1 / 2}
$$

Combination of eq 18 and 22 with eq 20 allows $\lambda$ to be calculated from data for $A, M_{\mathrm{L}}, X, M$, [ $\left.\eta\right]$, and $q$. In actual computations, $q_{\mathrm{BD}}$ and $M_{\mathrm{w}}$ were taken to be $q$ and $M$, respectively. The resulting $\lambda$ substituted into eq 18 and 22 gives $d_{\mathrm{h}}$ and $k$.

\section{APPLICATION TO LITERATURE DATA ON CELLULOSE DERIVATIVES}

By applying the Benoit-Doty, YamakawaStockmayer coil-limit, and Yamakawa-Fujii methods to the literature data, the wormlike chain parameters $q, \alpha_{\mathrm{s}, 3}^{w}, d_{\mathrm{h}}$, and $k$ were determined for cellulose/cadoxen, ${ }^{27}$ cellulose/iron sodium tartrate $(\mathrm{FeTNa}),{ }^{28} \mathrm{CA} \quad(2.92) / \mathrm{DMAc},{ }^{17}$ CA $\quad(2.46) /$ DMAc, ${ }^{19}$ CA (2.46)/acetone, ${ }^{16}$ CA (2.46)/tetrahydrofuran (THF), ${ }^{16} \mathrm{CA}(0.49) / \mathrm{DMAc},{ }^{18}$ cellulose nitrate (CN) (2.91)/acetone, ${ }^{12} \mathrm{CN}$ (2.55)/acetone, ${ }^{12}$ hydroxyethyl cellulose (HEC) (1)/water, ${ }^{29}$ ethyl hydroxyethyl cellulose (EHEC)/water, ${ }^{30}$ sodium carboxymethyl cellulose $(\mathrm{NaCMC})(0.88) / \mathrm{aq} \mathrm{NaCl}$ (at the limit of infinite ionic strength, i.e., $I \rightarrow \infty) .{ }^{31}$

\section{Cellulose}

Figure $2 \mathrm{a}$ and $2 \mathrm{~b}$ illustrate $q_{\mathrm{BD}}, q_{\mathrm{BD}}^{0}, q_{\mathrm{CL}}^{0}$, and $q_{\mathrm{YF}}$ for cellulose/cadoxen and cellulose/FeTNa. For the former system, all these $q$ are practically independent of $M_{w}$ and the following relation holds: $q_{\mathrm{BD}}>$ $q_{\mathrm{BD}}^{0} \simeq q_{\mathrm{CL}}^{0} \simeq q_{\mathrm{YF}}$. This figure contains no data of $q_{\mathrm{BD}}^{0}$

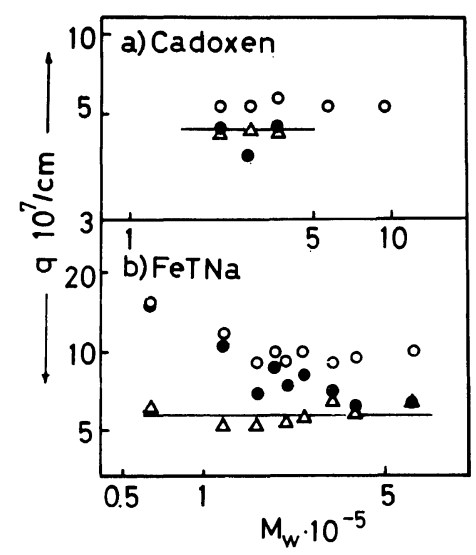

Figure 2. Various persistence lengths $q$ as a function of the weight-average molecular weight $M_{w}$ for cellulose/ cadoxen (a) and cellulose/FeTNa (b), O, $q_{\mathrm{BD}}$;,$q_{\mathrm{BD}}^{0}$; $\triangle, q_{\mathrm{YF}} ;$ solid line, $q_{\mathrm{CL}}^{0}$. 
and $q_{\mathrm{YF}}$ for two high molecular weight samples, because these samples did not allow $d_{\mathrm{h}}$ to be determined owing to $X=\infty$. For the latter system, $q_{\mathrm{BD}}$ and $q_{\mathrm{BD}}^{0}$ decrease with $M_{w}$, approaching an asymptotic value $\left(9.4 \times 10^{-7} \mathrm{~cm}\right.$ for $\left.q_{\mathrm{BD}}\right)$ at $M_{w}$ of $2 \times 10^{5}-4 \times 10^{5}$, and $q_{\mathrm{YF}}$ is constant, being smaller by $c a .3 .5 \times 10^{-7} \mathrm{~cm}$ than $q_{\mathrm{BD}}$ in the same solvent.

The significant difference between $q_{\mathrm{BD}}$ and $q_{\mathrm{BD}}^{0}$ for cellulose/cadoxen and that at high $M_{w}$ for cellulose/FeTNa reflect appreciable excludedvolume effects.

$q_{\mathrm{CL}}^{0}$ is approximately equivalent to $q_{\mathrm{BD}}^{0}$ over the whole range of $M_{w}$ in cadoxen or at high $M_{w}$ in FeTNa. At least, in these ranges of $M_{w}$ the Gaussian approximation is reasonable.

The $q$ values determined by the same method were found to be larger in FeTNa than in cadoxen. However, it would be unreasonable to regard this difference as significant, because cellulose is expectèd to form complexes with solvent, ${ }^{32}$ though no detailed structure of the complexes is as yet known. Thus, it is hazardous to conclude that cellulose is more rigid in FeTNa than in cadoxen.

\section{Cellulose Acetate}

Figure 3 depicts various $q$ plotted against $M_{w}$ for CA (2.92)/DMAc. $q_{\mathrm{BD}}, q_{\mathrm{BD}}^{0}$, and $q_{\mathrm{YF}}$ are practically independent of $M_{w} . q_{\mathrm{BD}}$ is $10 \%$ larger than $q_{\mathrm{BD}}^{0}$, indicating that the excluded-volume effect $\left(\alpha_{\mathrm{s}, 3}^{w} \simeq 1.1\right)$ on $q$ cannot be neglected. $q_{\mathrm{YF}}$ is smaller than $q_{\mathrm{BD}}$, as is the case of cellulose/cadoxen and FeTNa.

Assuming that the deviation from the linear relation between $[\eta] / M_{w}{ }^{1 / 2}$ and $M_{w}{ }^{1 / 2}$ reflects the semiflexible nature of CA, Dondos et al..$^{33}$ estimated for CA (2.92)/DMAc to be $4.1 \times 10^{-7} \mathrm{~cm}$ which is much smaller than those $\left(\geq 6.0 \times 10^{-7} \mathrm{~cm}\right)$ obtained in this paper. As Kamide et al..$^{1,16-19,34-36}$ pointed out, the Stockmayer-Fixman method underestimates the unperturbed chain dimensions if the draining effect is not negligible, as is the case for the systems examined here.

Figures $4 \mathrm{a}-4 \mathrm{c}$ show plots of $q_{\mathrm{BD}}, q_{\mathrm{BD}}^{0}, q_{\mathrm{CL}}^{0}$, and $q_{\mathrm{YF}}$ as a function of $M_{w}$ for CA (2.46) in DMAc (a), acetone (b), and THF (c). In DMAc and THF, any $q$ is independent of $M_{w}$ and $q_{\mathrm{BD}}>q_{\mathrm{BD}}^{0} \geq q_{\mathrm{CL}}^{0}$ (in both solvents) $\gg q_{\mathrm{YF}}$ (in DMAc). In contrast, in acetone, $q_{\mathrm{BD}}$ and $q_{\mathrm{BD}}^{0}$ decrease significantly with $M_{w}$. Kamide et al. ${ }^{16,19}$ indicated that CA (2.46) is non-Gaussian in acetone (i.e., $\left.a_{2}\left(\equiv \mathrm{d} \ln \left(\left\langle S^{2}\right\rangle_{0} / M\right) / \mathrm{d} \ln M\right)<0\right)$

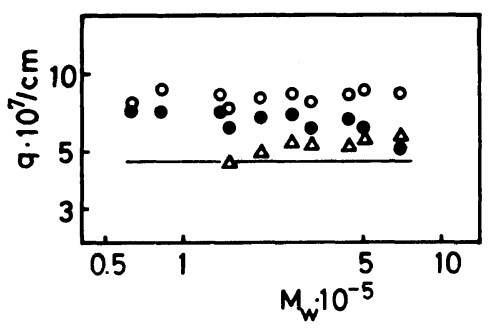

Figure 3. Various persistence lengths $q$ as a function of the weight-average molecular weight $M_{w}$ for CA (2.92)/ DMAc. $\bigcirc, q_{\mathrm{BD}} ; \bigcirc, q_{\mathrm{BD}}^{0} ; \triangle, q_{\mathrm{YF}}$; solid line, $q_{\mathrm{CL}}^{0}$.

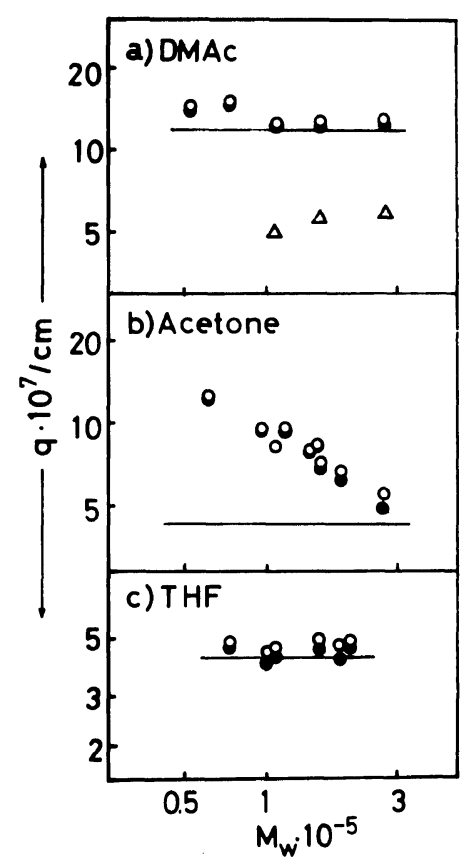

Figure 4. Various persistence lengths $q$ as a function of the weight-average molecular weight $M_{w}$ for CA (2.46)/ DMAc (a), acetone (b), and THF (c). $\bigcirc, q_{\mathrm{BD}} ; \bigcirc, q_{\mathrm{BD}}^{0} ; \triangle$, $q_{\mathrm{YF}}$; solid line, $q_{\mathrm{CL}}^{0}$.

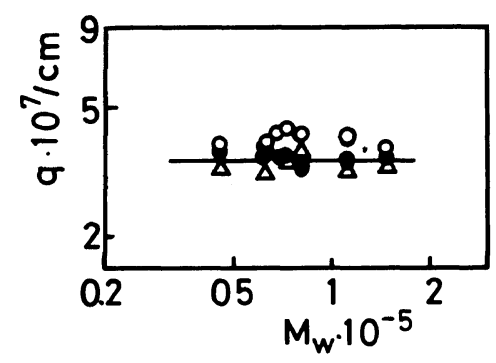

Figure 5. Various persistence lengths $q$ as a function of the weight-average molecular weight $M_{w}$ for CA (0.49)/ DMAc. $\bigcirc, q_{\mathrm{BD}} ; \bigcirc, q_{\mathrm{BD}}^{0} ; \triangle, q_{\mathrm{YF}}$; solid line, $q_{\mathrm{CL}}^{0}$. 
and Gaussian in DMAc and THF. In these three solvents, the excluded-volume effect on $q$ is only minor and, in fact, $\alpha_{\mathrm{s}, 3}^{\mathrm{w}}$ are less than 1.06 (see Figure 9).

Figure 5 shows plots of $q_{\mathrm{BD}}, q_{\mathrm{BD}}^{0}, q_{\mathrm{CL}}^{0}$, and $q_{\mathrm{YF}} v s$. $M_{w}$ for CA (0.49) in DMAc. All these $q$ are more or less independent of $M_{w}$, being smaller than $5 \times 10^{-7}$ $\mathrm{cm}$ and, the order: $q_{\mathrm{BD}}>q_{\mathrm{BD}}^{0} \simeq q_{\mathrm{YF}} \simeq q_{\mathrm{CL}}^{0}$. The equality of $q_{\mathrm{BD}}^{0}$ and $q_{\mathrm{CL}}^{0}$ suggests that the CA( $(0.49)$ chain in DMAc can be regarded as Gaussian.

\section{Cellulose Nitrate}

Figures $6 \mathrm{a}$ and $6 \mathrm{~b}$ show the plots of various $q v s$. $M_{w}$ for $\mathrm{CN}$ (2.91) and CN (2.55)/acetone, respectively. Except for the lowest molecular weight fraction of $\mathrm{CN}$ (2.91), $q_{\mathrm{BD}}$ and $q_{\mathrm{BD}}^{0}$ are nearly independent of $M_{w}$. In contrast, for $M_{w}>7 \times 10^{6}$, $q_{\mathrm{YF}}$ increases remarkably with $M_{w}$. This unexpected increase in $q_{\mathrm{YF}}$ cannot be explained by the excludedvolume effect alone. Throughout the entire range of $M_{w}$, the following relation was obtained: $q_{\mathrm{BD}} \geq$ $q_{\mathrm{BD}}^{0} \geq q_{\mathrm{CL}}^{0} \geq q_{\mathrm{YF}}$. In particular, $q_{\mathrm{BD}} \simeq q_{\mathrm{BD}}^{0}$ at low $M_{w}$ and $q_{\mathrm{BD}}^{0} \simeq q_{\mathrm{CL}}^{0}$ at high $M_{w}$. For relatively large molecular weights $\left(M_{w} \geq 7 \times 10^{6}\right), q_{\mathrm{YF}}$ tends to agree with other kinds of $q$.

The $q$ values of $\mathrm{CN}$ are smaller for lower DS, as was found in the case for $\sigma$ and $C_{\infty}$ in ref 1 .

\section{Miscellanea}

Figures $7 \mathrm{a}-7 \mathrm{c}$ illustrate the molecular weight dependence of $q$ for HEC/water, EHEC/water, and $\mathrm{NaCMC} /$ aq $\mathrm{NaCl}(I \rightarrow \infty)$, respectively. In the first two systems, $q_{\mathrm{BD}}$ and $q_{\mathrm{BD}}^{0}$ agree well with each other. Interestingly, for $\mathrm{HEC} /$ water, both $q_{\mathrm{BD}}$ and $q_{\mathrm{BD}}^{0}$ decrease significantly with increasing $M_{w}$. Kamide and Miyazaki ${ }^{1}$ pointed out that these two systems have negative $a_{2}$ (i.e., $a_{2}=-0.128$ for $\mathrm{HEC} /$ water and -0.256 for EHEC/water). A negative $a_{2}$ is considered due to a molecular weight dependence of the solvation of HEC or EHEC with water as a result of the large polarity of water. $q_{\mathrm{YF}}$ of $\mathrm{HEC} /$ water and EHEC/water are smaller than other kinds of $q$ and increase gradually with $M_{w}$, approaching $q_{\mathrm{BD}}$ or $q_{\mathrm{BD}}^{0}$ at high molecular weight.

$\mathrm{Aq} \mathrm{NaCl}(I \rightarrow \infty)$ is a Flory theta solvent for $\mathrm{NaCMC}^{31}$ (i.e., $q_{\mathrm{BD}}=q_{\mathrm{BD}}^{0}$ ). The $q$ values of $\mathrm{NaCMC}$ in this solvent are about $5.0 \times 10^{-7} \mathrm{~cm}$, indicating that $\mathrm{NaCMC}$ in aq $\mathrm{NaCl}(I \rightarrow \infty)$ is flexible.

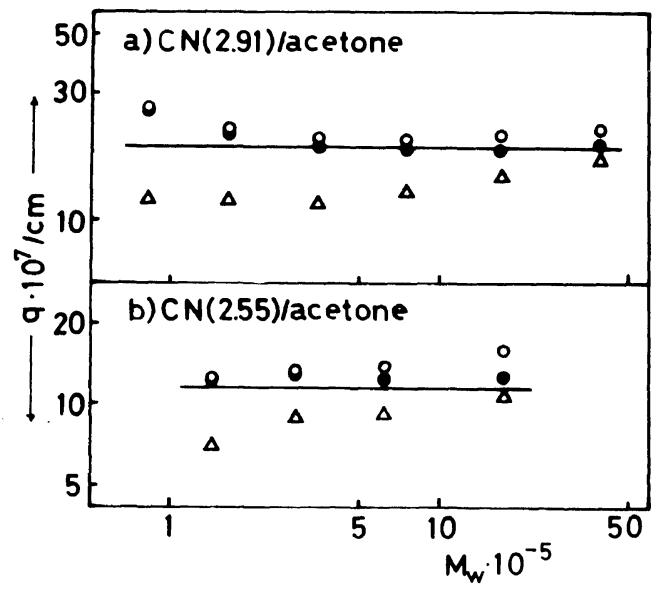

Figure 6. Various persistence lengths $q$ as a function of the weight-average molecular weight $M_{w}$ for $\mathrm{CN}$ (2.91)/ acetone (a) and $\mathrm{CN}$ (2.55)/acetone (b). $\bigcirc, q_{\mathrm{BD}} ; \boldsymbol{O}, q_{\mathrm{BD}}^{0}$; $\triangle, q_{\mathrm{YF}} ;$ solid line, $q_{\mathrm{CL}}^{0}$.

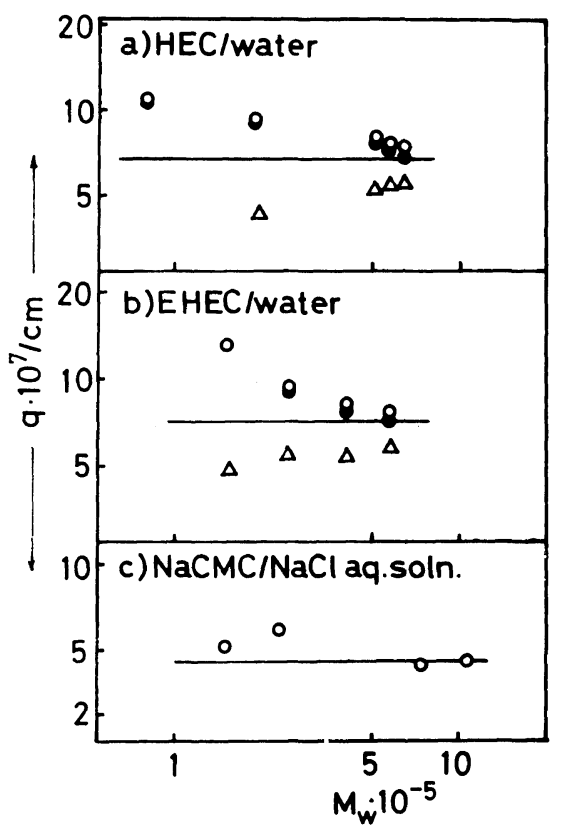

Figure 7. Various persistence lengths $q$ as a function of the weight-average molecular weight $M_{w}$ for $\mathrm{HEC} /$ water (a), EHEC/water (b), and $\mathrm{NaCMC} / \mathrm{NaCl}$ aq soln. (c). $\bigcirc$, $q_{\mathrm{BD}} ; 0, q_{\mathrm{BD}}^{0} ; \triangle, q_{\mathrm{YF}} ;$ solid line, $q_{\mathrm{CL}}^{0}$.

\section{DISCUSSION}

Table I summarizes the averages and standard deviations of $d_{\mathrm{h}}, \lambda$, and $k$ for cellulose and its 
Table I. Averages and standard deviations of the hydrodynamic diameter $d_{\mathrm{h}}$, the proportionality constant $\lambda$, and the hydrodynamic interaction parameters $k$ of cellulose and its derivatives/solvent systems

\begin{tabular}{|c|c|c|c|c|c|}
\hline $\begin{array}{l}\text { Polymer } \\
(\mathrm{DS})^{\mathrm{a}}\end{array}$ & Solvent & $\begin{array}{c}\text { Sample } \\
\text { No. }\end{array}$ & $\frac{d_{\mathrm{h}}}{10^{-7} \mathrm{~cm}}$ & $\lambda$ & $k$ \\
\hline \multirow[t]{2}{*}{ Cellulose $(0)$} & Cadoxen & 3 & $1.6\left(0.4_{5}\right)^{\mathrm{b}}$ & $0.50\left(0.1_{4}\right)^{b}$ & $0.15\left(0.0_{3}\right)^{b}$ \\
\hline & $\mathrm{FeTNa}^{\mathrm{c}}$ & 9 & $2.8\left(0.8_{4}\right)$ & $0.71\left(0.2_{0}\right)$ & $0.08\left(0.0_{2}\right)$ \\
\hline \multirow[t]{2}{*}{ CA (2.92) } & $\mathrm{DMAc}^{\mathrm{d}}$ & 10 & $3.4\left(0.4_{8}\right)$ & $0.72\left(0.1_{1}\right)$ & $0.16\left(0.0_{3}\right)$ \\
\hline & DMAc & 5 & $2.8\left(0.5_{4}\right)$ & $0.50\left(0.0_{0}\right)$ & $0.07\left(0.0_{1}\right)$ \\
\hline \multirow[t]{2}{*}{$\mathrm{CA}(2.46)$} & Acetone & 9 & $3.6\left(0.3_{5}\right)$ & $0.72\left(0.0_{9}\right)$ & $0.13\left(0.0_{4}\right)$ \\
\hline & $\mathrm{THF}^{\mathrm{e}}$ & 6 & $3.9\left(0.5_{0}\right)$ & $0.43\left(0.0_{5}\right)$ & $0.38\left(0.0_{4}\right)$ \\
\hline CA (0.49) & DMAc & 10 & $2.4\left(0.5_{0}\right)$ & $0.40\left(0.1_{1}\right)$ & $0.35\left(0.0_{5}\right)$ \\
\hline $\mathrm{CN}(2.91)$ & Acetone & 6 & $2.6\left(1.2_{9}\right)$ & $0.50\left(0.0_{0}\right)$ & $0.11\left(0.0_{2}\right)$ \\
\hline $\mathrm{CN}(2.55)$ & Acetone & 4 & $2.1\left(0.3_{1}\right)$ & $0.35\left(0.0_{5}\right)$ & $0.10\left(0.0_{1}\right)$ \\
\hline HEC (1) & Water & 5 & $2.9\left(0.7_{6}\right)$ & $0.76\left(0.0_{5}\right)$ & $0.10\left(0.0_{3}\right)$ \\
\hline EHEC (2) & Water & 4 & $2.9\left(0.2_{7}\right)$ & $0.63\left(0.1_{1}\right)$ & $0.10\left(0.0_{4}\right)$ \\
\hline $\mathrm{NaCMC}(0.88)$ & $\begin{array}{l}\text { Aq sodium chloride } \\
\left(I^{\mathrm{f}} \rightarrow \infty\right)\end{array}$ & 4 & $3.0\left(1.2_{0}\right)$ & $0.25\left(0.1_{5}\right)$ & $0.53\left(0.2_{5}\right)$ \\
\hline
\end{tabular}

\footnotetext{
a Degree of substitution.

b Standard deviation.

c Iron sodium tartrate.

d $N, N$-Dimethylacetamide.

e Tetrahydrofuran.

f Ionic strength.
}

derivatives analyzed above. The average $d_{\mathrm{h}}$ lies between $1.6 \times 10^{-7}$ and $3.9 \times 10^{-7} \mathrm{~cm}$. These values are apparently larger than those estimated from crystallographic data, which are $9.0 \times 10^{-8} \mathrm{~cm}$ and $8.2 \times 10^{-7} \mathrm{~cm}$, respectively, when the a and $\mathrm{b}$ axes of a cellulose II unit cell are taken for the diameters. ${ }^{14}$ The solvent layer formed around pyranose rings should increase the radius of a hydrodynamic segment. However, there is available no direct estimation of $d_{\mathrm{h}}$ for solvated cellulose molecules. Most of the average $\lambda$ are smaller than 0.8 , indicating that the hydrodynamic segment length is smaller than the statistical segment length. The average values of $k$ for all cellulose derivatives examined are significantly smaller than the value at the nondraining limit $(k \geq 10){ }^{7}$ so that the hydrodynamic interaction should be relatively weak in cellulose and its derivatives in solution.

Figure 8 shows the relation between $\alpha_{\mathrm{s}, 3}^{w}$ and $d_{\mathrm{h}}$ for a CA (2.46) sample (EF3-15) in DMAc. ${ }^{19}$ In the range $d_{\mathrm{h}} \geq 5 \times 10^{-8} \mathrm{~cm}, \alpha_{\mathrm{s}, 3}^{w}$ is almost independent of $d_{\mathrm{h}}$. As shown in Table $\mathrm{I}$, the $d_{\mathrm{h}}$ values of cellulose derivatives are larger than $5 \times 10^{-8} \mathrm{~cm}$. Even if these $d_{\mathrm{h}}$ values are not very accurate, it may be

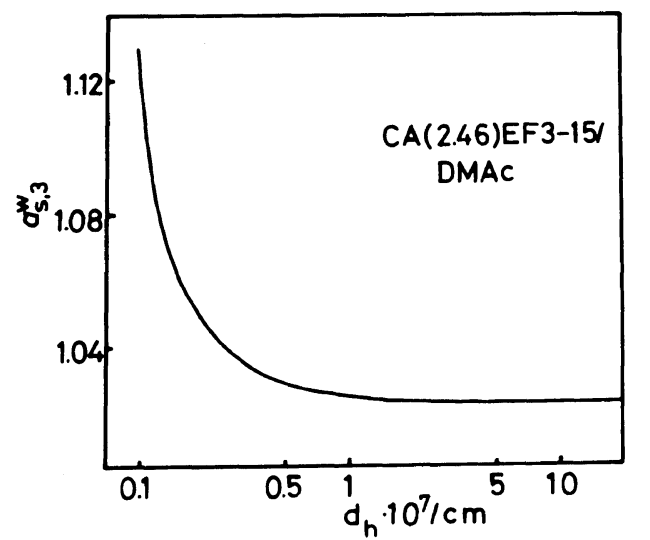

Figure 8. Relation between the expansion factor $\alpha_{s, 3}^{w}$ and the hydrodynamic diameter $d_{\mathrm{h}}$ for a CA (2.46) sample (code EF3-15 19 ) in DMAc.

concluded that $\alpha_{\mathrm{s}, 3}^{w}$ is insensitive to $d_{\mathrm{h}}$.

Figure 9 shows $\alpha_{\mathrm{s}, 3}^{w}$ plotted against $\alpha_{\mathrm{s}, 3}^{p}$, with the solid line representing the relation $\alpha_{s, 3}^{w}=\alpha_{s, 3}^{p}$. For all the polymer samples investigated, the relation $\alpha_{\mathrm{s}, 3}^{w}<\alpha_{\mathrm{s}, 3}^{p}$ holds. In other words, the analysis in terms of the wormlike chain model results in a 


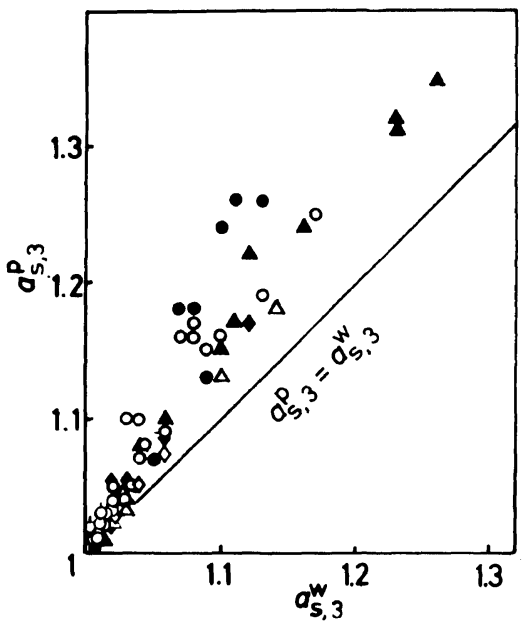

Figure 9. The plot of $\alpha_{s, 3}^{w}$ and $\alpha_{s, 3}^{p} . \triangle$, cellulose/ cadoxen; $\Delta$, cellulose/FeTNa; $\bigcirc$, CA (2.92)/DMAc; $O$, CA (2.46)/DMAc; - $\bigcirc$, CA (2.46)/acetone; O-, CA (2.46)/THF;, $\mathrm{CA}(0.49) / \mathrm{DMAc} ; \diamond, \mathrm{CN}$ (2.91)/acetone; $\diamond, \mathrm{CN}$ (2.55)/acetone; $\square$, HEC/water; $\square, \mathrm{EHEC} /$ water; $\nabla, \mathrm{NaCMC} / \mathrm{aq} \mathrm{NaCl}$. The solid line represents the relation $\alpha_{s, 3}^{w}=\alpha_{s, 3}^{p}$.

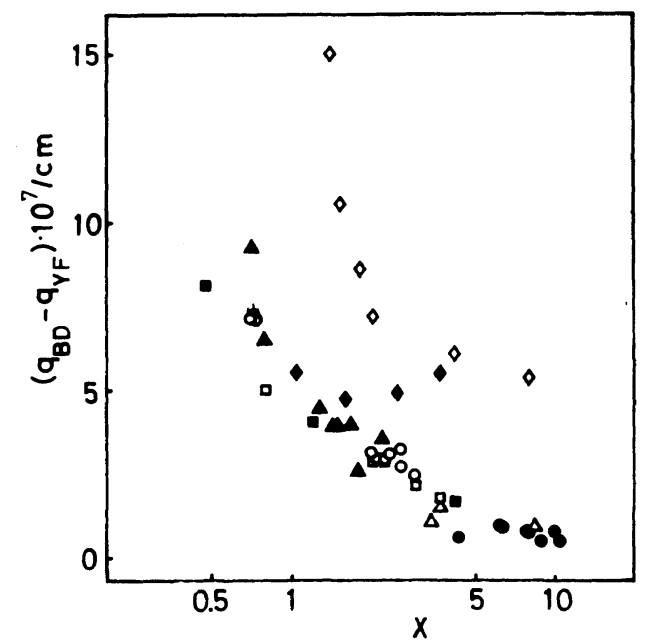

Figure 10. The difference between the Benoit-Doty persistence length $q_{\mathrm{BD}}$ and the Yamakawa-Fujii persistence length $q_{\mathrm{YF}}, q_{\mathrm{BD}}-q_{\mathrm{YF}}$, plotted against the draining parameter $X$ for cellulose and its derivatives in solution. The symbols in the figure are the same as in Figure 9.

smaller expansion factor than does that in terms of the pearl necklace model. The $\alpha_{s, 3}^{w}$ values for cellulose derivatives are smaller than 1.14 , except the

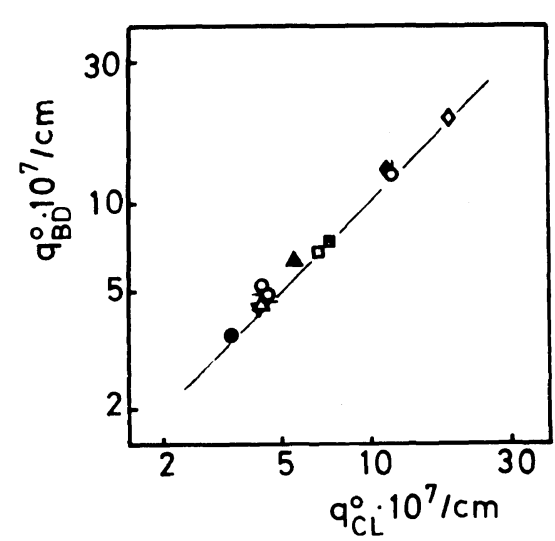

Figure 11. Correlation between the unperturbed Benoit-Doty persistence length $q_{\mathrm{BD}}^{0}$ and the persistence length at the coil limit $q_{\mathrm{CL}}^{0}$. The symbols are the same as in Figure 9.

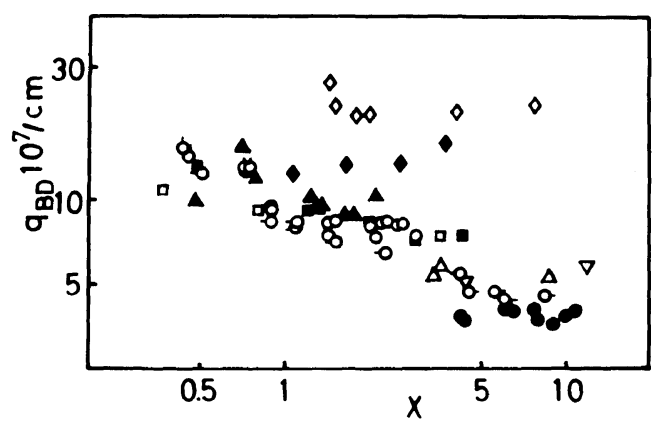

Figure 12. The dependence of the Benoit-Doty persistence length $q_{\mathrm{BD}}$ on the draining parameter $X$. The symbols are the same as in Figure 9.

high molecular weight samples in cellulose $/ \mathrm{FeTNa}$, CA (2.92)/DMAc, and CA (0.49)/DMAc. Thus, the solvents may be considered to be poor. Therefore, the conclusions on the thermodynamic properties of cellulose derivative solutions deduced by Kamide and his coworkers ${ }^{1,16-19}$ from the pearl necklace model are acceptable.

As shown in the previous section, $q_{\mathrm{BD}}$ does not agree closely with $q_{\mathrm{YF}}$ for any cellulose drivatives examined. Figure 10 shows the difference $q_{\mathrm{BD}}-q_{\mathrm{YF}}$ plotted against the draining parameter $X$ estimated by method $1 \mathrm{~A} .{ }^{1}$ Evidently, for any of these cellulose derivatives $q_{\mathrm{BD}}>q_{\mathrm{YF}}$ holds and $q_{\mathrm{BD}}-q_{\mathrm{YF}}$ has a negative correlation with $X$ (correlation coefficient $r=-0.60$ ). Considering that $q_{\mathrm{YF}}$ is less dependent on $d_{\mathrm{h}}$ for $d_{\mathrm{h}}>5 \times 10^{-8} \mathrm{~cm},{ }^{10}$ we can conclude that the relation $q_{\mathrm{BD}}>q_{\mathrm{YF}}$ is due neither to a relatively 
Table II. Persistence length of stiff and flexible polymers estimated by various methods

\begin{tabular}{|c|c|c|c|c|}
\hline Polymer & Solvent & $\frac{q \times 10^{8}}{\mathrm{~cm}}$ & Method & ref \\
\hline Poly(vinylpyridiniumbutylbromide) & $5 \times 10^{-4} \mathrm{M}$ Aq sodium chloride & 704 & V & 7 \\
\hline Polymethylacrylic acid & $5 \times 10^{-4} \mathrm{M}$ Aq sodium chloride & 625 & V & 7 \\
\hline \multirow[t]{2}{*}{ DNA $^{b}$} & $0.79 \mathrm{M}$ Aq sodium chloride & 520 & $\mathrm{~V}$ & 7 \\
\hline & $0.2 \mathrm{M} \mathrm{Aq}$ sodium chloride & 565 & $\mathrm{~V}$ & 10 \\
\hline Poly(hexyl isocyanate) & $n$-Hexane & 420 & LS, V, s & 11 \\
\hline Poly( $n$-butylisocyanate) & Tetrachloromethane & 375 & $\mathrm{~V}$ & 10 \\
\hline Polymethylmethacrylate & Benzene & 14 & SAXS & 38 \\
\hline Polyethylene & Bis-2-ethylhexyl adipate & $9.6^{\mathrm{a}}$ & LS & 39 \\
\hline Polyvinylacetate & Heptane/3-methyl-2-butanone & $16^{\mathrm{a}}$ & LS & 40 \\
\hline Polystyrene & Cyclohexane & $9-10$ & SAXS & 41 \\
\hline
\end{tabular}

a Estimated from unperturbed chain dimensions reported in the literature.

b DNA, deoxyribonucleic acid.

inaccurate $d_{h}$ value nor to the polydispersity of the samples, but to a significant role played by the free draining effect. This is because the correction of $[\eta]$ in the YF theory for this effect should raise the value of $q_{\mathrm{YF}}$.

Yamakawa and Fujii ${ }^{10}$ found that the draining term does not appear in the integral equation for the flow rate of fluid, provided that the fluid does not slip on the surface of the wormlike cylinder, and concluded that the draining effect does not exist in the wormlike cylinder model. As has often been mentioned, in cellulose derivative solutions, a solvated solvent layer is formed around the naked cellulose chain. Obviously, since these systems have not distinct boundary between the solvent as a continuous medium and the solute molecule, the boundary condition employed by Yamakawa and Fujii seems to be too oversimplified to explain the dilute solution properties of cellulose derivatives.

Figure 11 shows the relation between $q_{\mathrm{BD}}^{0}$ and $q_{\mathrm{CL}}^{0}$ for the highest molecular weight sample of each cellulose derivative. $q_{\mathrm{BD}}^{0}$ agrees well with $q_{\mathrm{CL}}^{0}$ for any of the cellulose derivatives investigated, implying that $A$ in the pearl necklace model varies with $q$ in the wormlike chain model at these highest molecular weight samples.

Figure 12 shows the relationship between $q_{\mathrm{BD}}$ and $X$. Except for cellulose nitrate (2.91 and 2.55)/ acetone, $q_{\mathrm{BD}}$ decreases significantly with an increase in $X$, indicating that the cellulose derivative chain becomes less flexible as the draining effect plays an important role. Recently, Tsvetkov and Andreeva ${ }^{37}$ stated that the hydrodynamic interaction is weaker for rigid chain coils than flexible chain coils and that the draining effect should be taken into the hydrodynamic parameters. However, they did not investigate quantitatively the role of the draining effect.

Since, as shown above, the excluded-volume effect is small, though not negligible, and the draining effect plays an important role in solution of cellulose derivatives, $q_{\mathrm{BD}}^{0}$ obtained from the light scattering data should be a more reliable measure of polymer chain rigidity than the hydrodynamic persistence length including $q_{\mathrm{YF}}$. The $q_{\mathrm{BD}}^{0}$ values of cellulose derivatives vary from $3.0 \times 10^{-7}$ to $2.5 \times 10^{-6} \mathrm{~cm}$, with most found between $3.0 \times 10^{-7}$ $\mathrm{cm}$ and $8.0 \times 10^{-7} \mathrm{~cm}$ (see Figures 2-7). In Table II, the literature $q$ values obtained by analyzing light scattering (LS), viscosity (V), sedimentation (s), and small-angle $X$ ray scattering (SAXS) data are given. Although the $q$ values of typical stiff chains, such as DNA and poly(hexyl isocyanate), are not corrected for the polydispersity or excluded-volume effects, these $q$ values are five to ten times larger than those of cellulose derivatives. For flexible polymers like polystyrene, $q$ is always less than about $2.0 \times 10^{-7}$ $\mathrm{cm}$. Thus, it is clear that the chains of cellulose derivatives may be classified as semiflexible.

In Figure $13, q_{\mathrm{BD}}^{0}$ is plotted against the chemical shifts of $O$-acetyl methyl and hydroxyl groups $\left(\delta_{\mathrm{CH}_{3}}\right.$ and $\left.\delta_{\mathrm{OH}}\right)^{42}$ and the number of grams $(n)^{43}$ of solvent 


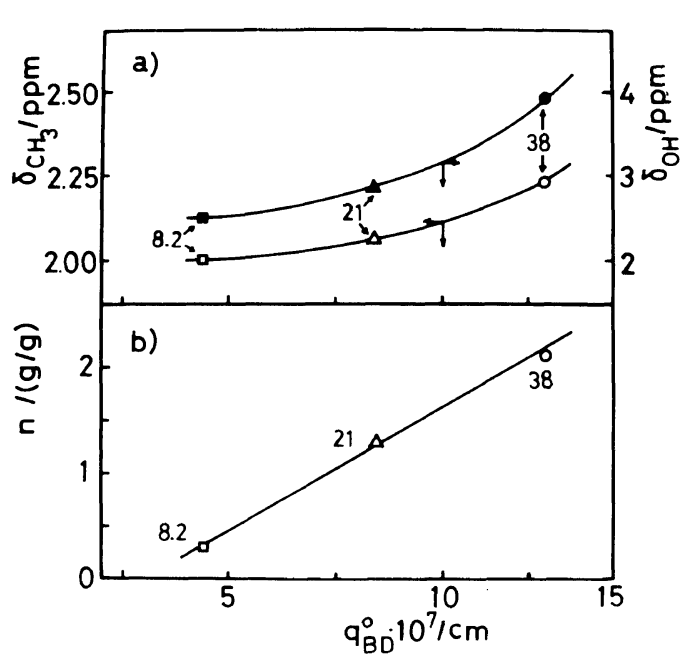

Figure 13. a) Plot of the chemical shifts of methyl and hydroxyl protons, $\delta_{\mathrm{CH}_{3}}$ (open marks) and $\delta_{\mathrm{OH}}$ (closed marks) against the unperturbed Benoit-Doty persistence length $q_{\mathrm{BD}}^{0}$ for a CA (2.46) sample (code EF3-13 $13^{16,19}$ ) in DMAc (circle), acetone (triangle), and THF (rectangle). b) Plot of the number grams $n$ of solvated molecules per $1 \mathrm{~g}$ of polymer molecules against $q_{\mathrm{BD}}^{0}$ for CA (2.46) in three solvents.

molecules per $1 \mathrm{~g}$ of polymer in CA (2.46)/DMAc, acetone, and THF. The figures appearing on the curves indicate the dielectric constant of the solvents. It can be seen that $q_{\mathrm{BD}}^{0}$ is correlated closely with the degree of solvation, being larger in a more polar solvent. In a polar solvent, probably there is a strong interaction between the $O$-acetyl methyl and hydroxyl groups and the solvent and the solvent molecules about the polymer chain interfere with the internal rotation of the cellulose acetate chain. These should lead to an expanded conformation of the chain. The solvation was also observed for other cellulose derivatives, ${ }^{44}$ and we conclude that it contributes greatly to the rigidity of the cellulose chain.

\section{CONCLUSIONS}

(1) Cellulose and its derivatives belong to the category of semiflexible polymers and not to the family of inflexible polymers.

(2) Cellulose and its derivatives in solution can be reasonably well modeled either by the pearl necklace chain or the wormlike chain, and the conclusions deduced on the basis of the pearl necklace model are definitely acceptable.

(3) The excluded-volume effect is relatively small in solutions of cellulose and its derivatives, but cannot be ignored when estimating the unperturbed persistence length.

(4) The draining effect cannot be neglected even in the wormlike chain model. This effect is real, not the apparent one found by applying the experimental data for cellulose and its derivatives beyond the applicability limit of the pearl necklace model.

(5) The unperturbed persistence length $\left(q_{\mathrm{BD}}^{0}\right)$, evaluated by the BD theory, is the most reliable among $q$ estimated by various methods.

Acknowledgements. The author should like to express his most sincere gratitude to Dr. Kenji Kamide, General Maneger of Textile Research Laboratory, Asahi Chemical Industry Co. Ltd., for his guidance and stimulating comments and to Dr. Hidematsu Suzuki, Institute for Chemical Research, Kyoto University for his remarks on the manuscript.

\section{REFERENCES}

1. K. Kamide and Y. Miyazaki, Polym. J., 10, 409 (1978).

2. O. Kratky and G. Porod, Recl. Trav. Chim., 68, 1106 (1949).

3. H. Benoit and P. M. Doty, J. Phys. Chem., 57, 958 (1953).

4. J. E. Hearst, J. Chem. Phys., 40, 1506 (1964).

5. A. Peterlin, J. Polym. Sci., 8, 173 (1952).

6. J. E. Hearst and W. H. Stockmayer, J. Chem. Phys., 37, 1425 (1962).

7. R. Ullman, J. Chem. Phys., 49, 5486 (1968).

8. R. Ullman, J. Chem. Phys., 53, 1734 (1970).

9. H. Yamakawa and M. Fujii, Macromolecules, 6, 407 (1973).

10. H. Yamakawa and M. Fujii, Macromolecules, 7, 128 (1974).

11. H. Murakami, T. Norisuye, and H. Fujita, Macromolecules, 13, 345 (1980).

12. E. Penzel and G. V. Schulz, Makromol. Chem., 113, 64 (1968).

13. H. Maeda, N. Saito, and W. H. Stockmayer, Polym. J., 2, 94 (1971).

14. D. W. Jones, "Cellulose and Cellulose Derivatives," N. M. Bikales and L. Segal, Ed., Wiley-Interscience, New York, N. Y., 1971, p 127.

15. H. Yamakawa and W. H. Stockmayer, J. Chem. Phys., 57, 2843 (1972).

16. K. Kamide, Y. Miyazaki, and T. Terakawa, Polym. 
J., 11, 285 (1979)

17. K. Kamide, Y. Miyazaki, and T. Abe, Polym. J., 11, 523 (1979)

18. K. Kamide, M. Saito, and T. Abe, Polym. J., 13, 421 (1981).

19. K. Kamide and M. Saito, Polym. J., 14, 517 (1982).

20. M. Fixman, J. Chem. Phys., 36, 3123 (1962).

21. K. Kamide and M. Saito, Eur. Polym. J., in press.

22. M. Kurata, M. Fukatsu, H. Sotobayashi, and H. Yamakawa, J. Chem. Phys., 41, 139 (1964).

23. J. Kirkwood and J. Riseman, J. Chem. Phys., 16, 565 (1948).

24. E. V. Korneeva, et al., Vysokomol. Soedin, Ser. A, 21, 1547 (1979).

25. M. Kurata and H. Yamakawa, J. Chem. Phys., 29, 311 (1958).

26. For example, eq B3 and B4 in ref 7 should be read as eq $\mathrm{R} 1$ and $\mathrm{R} 2$, respectively.

$A_{i j}=\frac{k L W_{j}}{2 q} K\left(\frac{L}{2 q}\left|x_{j}-x_{i}\right|\right) \quad(i \neq j)$

and

$$
\begin{aligned}
A_{i i}=1+ & \frac{W_{i} k L}{q\left(x_{i+1}-x_{i-1}\right)} \int_{\left(x_{i-1}+x_{i}\right) / 2}^{\left(x_{i+1}+x_{i-1}\right) / 2} \\
& \times K\left(\frac{L}{2 q}\left|x-x_{i}\right|\right) \mathrm{d} x \quad(i=j)
\end{aligned}
$$

Equation R2 can be rearranged to eq R3 using the $m$ th Gaussian Quadrature (see text).

$$
\begin{aligned}
A_{i i}=1 & +\frac{W_{i} k L}{4 q} \sum_{j=1}^{m} W_{j} K \\
& \times\left[\left(\frac{q L}{8}\right)\left|\left(x_{i+1}-x_{i-1}\right) x_{j}+\left(x_{i+1}-2 x_{i}+x_{i-1}\right)\right|\right]
\end{aligned}
$$

27. D. Henley, Ark. Kemi., 18, 327 (1961).

28. L. Valtasaari, Makromol. Chem., 150, 117 (1971).

29. W. Brown and D. Henley, Makromol. Chem., 108, 153 (1967).

30. R. S. Manley, Ark. Kemi., 9, 519 (1956).

31. W. Brown and D. Henley, Makromol. Chem., 79, 68 (1964).

32. G. Jayme, "Cellulose and Cellulose Derivatives," N. M. Bikales and L. Segal, Ed., Wiley-Interscience, New York, N. Y., 1971, p 381.

33. A. Dondos and G. Stakos, Eur. Polym. J., 16, 1215 (1980).

34. K. Kishino, T. Kawai, T. Nose, M. Saito, and K. Kamide, Eur. Polym. J., 17, 623 (1981).

35. K. Kamide and M. Saito, Eur. Polym. J., 17, 1049 (1981).

36. K. Kamide and M. Saito, Eur. Polym. J., 18, 661 (1982).

37. V. N. Tsvetkov and L. N. Andreeva, Adv. Polym. Sci., 39, 95 (1981).

38. O. Kriste and O. Kratky, X. Phys. Cem., 31, 363 (1962).

39. A. Kotera, H. Matsuda, and A. Wada, Rep. Prog. Polym. Phys., 8, 5 (1965).

40. A. R. Schulz, J. Am. Chem. Soc., 76, 3423 (1954).

41. Y. Wada and K. Okano, Rep. Prog. Polym. Phys., 7, 19 (1964).

42. K. Kamide, K. Okajima, and M. Saito, Polym. J., 13, 115 (1981).

43. K. Kamide and M. Saito, unpublished results.

44. see for example, A. Dobry, J. Chim. Phys., 35, 20 (1938); E. Calvet, Compt. Rend., 212, 542 (1941), 213, 126 (1942), 215, 138 (1942); W. R. Moore and B. M. Tidswell, Makromol. Chem., 81, 1 (1965); W. R. Moore, J. Polym. Sci., C, 16, 571 (1967); W. R. Moore, "Solution Properties of Natural Polymers: An International Symposium in Edinburgh," The Chemical Society Ed., The Chemical Society Burlington House, London, W.1, 1968, pp 185-194. 